\title{
Extremes of age are associated with differences in the expression of selected pattern recognition receptor genes and ACE2, the receptor for SARS-CoV-2: implications for the epidemiology of COVID-19 disease
}

\author{
Stephen W. Bickler ${ }^{1,2,3^{*}} \mathbb{E}$, David M. Cauvi ${ }^{2,3}$, Kathleen M. Fisch ${ }^{4}$, James M. Prieto ${ }^{1,5}$, Alicia G. Sykes ${ }^{1,5}$,

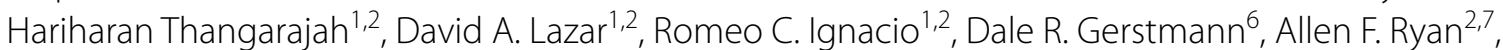 \\ Philip E. Bickler ${ }^{8}$ and Antonio De Maio ${ }^{2,3}$
}

\begin{abstract}
Background: Older aged adults and those with pre-existing conditions are at highest risk for severe COVID-19 associated outcomes.

Methods: Using a large dataset of genome-wide RNA-seq profiles derived from human dermal fibroblasts (GSE113957) we investigated whether age affects the expression of pattern recognition receptor (PRR) genes and ACE2, the receptor for SARS-CoV-2.
\end{abstract}

Results: Extremes of age are associated with increased expression of selected PRR genes, ACE2 and four genes that encode proteins that have been shown to interact with SAR2-CoV-2 proteins.

Conclusions: Assessment of PRR expression might provide a strategy for stratifying the risk of severe COVID-19 disease at both the individual and population levels.

Keywords: SARS-CoV-2, Pattern recognition receptors, Toll-like receptor 4, Aging, Skin fibroblasts

\section{Background}

Most people infected with SARS-CoV-2 will have mild to moderate cold and flu-like symptoms, or even be asymptomatic [1]. Older aged adults, and those with underlying conditions such as diabetes mellitus, chronic lung disease and cardiovascular disease are at highest risk for severe COVID-19 associated outcomes [2]. The highest case fatality rates are in the 80 years and older age

${ }^{*}$ Correspondence: sbickler@health.ucsd.edu

1 Rady Children's Hospital, Rady Children's Hospital, University of California

San Diego, 3030 Children's Way, San Diego, CA 92123, USA

Full list of author information is available at the end of the article group $(7.8 \%)$, with the lowest in the $0-9$ years age group $(0.00161 \%)$ [3]. Age greater than 80 years has more than twenty times the risk of COVID-19-related death compared to people aged 50-59 years [4]. The reasons for these markedly different outcomes at the extremes of age and for the occasional death that occurs in apparently healthy younger patients remain poorly understood.

Pattern recognition receptors (PRRs) play crucial roles in the innate immune response by recognizing pathogen-associated molecular patterns (PAMPs) and molecules derived from damaged cells, referred to as damage-associated molecular patterns (DAMPs) $[5,6]$. PRRs are coupled to intracellular signaling cascades that

(c) The Author(s) 2021. Open Access This article is licensed under a Creative Commons Attribution 4.0 International License, which permits use, sharing, adaptation, distribution and reproduction in any medium or format, as long as you give appropriate credit to the original author(s) and the source, provide a link to the Creative Commons licence, and indicate if changes were made. The images or other third party material in this article are included in the article's Creative Commons licence, unless indicated otherwise in a credit line to the material. If material is not included in the article's Creative Commons licence and your intended use is not permitted by statutory regulation or exceeds the permitted use, you will need to obtain permission directly from the copyright holder. To view a copy of this licence, visit http://creativecommons.org/licenses/by/4.0/. The Creative Commons Public Domain Dedication waiver (http://creativeco mmons.org/publicdomain/zero/1.0/) applies to the data made available in this article, unless otherwise stated in a credit line to the data. 
control transcription of a wide spectrum of inflammatory genes [7]. Humans have several distinct classes of PRRs, including Toll-like receptors (TLRs), NOD-like receptors (NLRs), RIG-like receptors (RLRs), C-type lectin receptors (CLRs) and intracellular DNA sensors [8]. PRRs play a critical role in the inflammatory response induced by viruses and are important determinants of outcome [9-12].

In this study, we examined whether extremes of age affect the expression of PPR genes, ACE2 and proteins that have been shown to interact with SARS-CoV-2. We found extremes of age are associated with differences in the expression of PRR genes, ACE2 and several genes that encode proteins known to interact with SAR2-CoV-2.

\section{Methods}

\section{Human dermal fibroblast dataset}

Our analysis was done using RNA-seq data (GSE113957) from the National Center for Biotechnology Information (NCBI, Bethesda, MD, USA). Normalized TMM gene counts per million for the individual dermal fibroblast cell lines were downloaded from the GEO RNA-seq Experiments Interactive Navigator (GREIN) [13, 14].

\section{Identification of differentially expressed genes and enrichment analysis}

Limma-Voom $[15,16]$ was used to identify differentially expressed genes between the oldest ( $\geq 80$ years, $N=33$ ) and youngest $(\leq 10$ years, $N=14$ ) age groups. Differentially expressed genes were defined as those with an Adjusted $\mathrm{P}$ value $<0.05$ after multiple testing correction and an absolute log2Fold Change $>1$.0. Enrichment analysis of the differentially expressed genes was performed with ToppGene [17].

\section{Correlation analysis}

Pairwise Pearson correlation coefficients were calculated between the normalized gene counts of the 21 PRR genes, ACE2 and age, over all 133 samples using GraphPad Prism version 8.0.

\section{Age related interactions with SARS-CoV-2 proteins}

Protein-protein interactions linking differentially expressed genes and SARS-CoV-2 proteins were identified by overlaying differentially expressed genes in the oldest and youngest age groups on to the SARS-CoV-2 human protein-protein interaction map reported by Gordon, et al. [18]. Network visualization was performed using Cytoscape [19] the NDEx v2.4.5 [20].

\section{Results}

Dermal fibroblast RNA-seq data set

Dermal fibroblast cultures retain age-dependent phenotypic, epigenomic, and transcriptomic changes [21-24]. As such, fibroblast cultures have been proposed as a model for studying aging and related diseases [25]. We leveraged this approach to investigate the affect aging has on PRR and ACE2 gene expression. For our analysis we used a large dataset of genome-wide RNA-seq profiles derived from human dermal fibroblasts (GSE $113,957)$ that was previously used to develop an ensemble machine learning method that could predict chronological age to a median error of 4 years [25]. The dataset includes samples from 133 "apparently healthy individuals" aged between 1 to 94 years. Given that COVID-19 disease has markedly different outcomes at the extremes of age, we first examined the gene expression differences between the oldest ( $\geq 80$ years, $N=33$ ) and the youngest ( $\leq 10$ years, $\mathrm{N}=14$ ) age groups (see "Methods" section). After filtering out genes with low expression (cpm $>0.5$ in at least two samples), a total of 1252 genes were differentially expressed between the oldest relative to the youngest age group (Fig. 1a, Additional file 1: Suppl Table 1a). Differentially expressed genes were enriched in KEGG pathways involved in Cell Cycle and DNA replication, among others (Fig. 1b, Additional file 2: Suppl Table 2).

\section{Age is associated with broad changes in PRR gene expression}

We next focused on whether the expression of individual PRR genes change with age. Between the oldest ( $\geq 80$ years) and the youngest ( $\leq 10$ years) age groups we found three differentially expressed PRR genes (TLR3, TLR4, and IHIF1) that had a $\log 2 \mathrm{FC}>1.0$ (Fig. 1c,d, Additional file 1: Suppl Table 1b). Age was correlated with the expression of 20 out of 21 PRR genes (Fig. 2a, Additional file 3, Suppl Table 3a-c). Normalized gene counts for TLR3, TLR4 and IHIF1 expressed as a function of age are shown in Fig. 2b. Of these, TLR4 had the greatest fold change increase $(\log 2 \mathrm{FC}=2.6)$ and the highest correlation coefficient with age (Pearson $\mathrm{r}$ 0.60, Adj. P Value 2.05E-14) (Additional files 1 and 3: Suppl Tables 1b and 3a-c). Plots of the other TLR genes counts are provided in Additional file 4: Suppl Figure 1.

The expression of two PRR genes were negatively correlated with age, Nucleotide-binding oligomerization domain-containing protein 1 (NOD1) $(\log 2 \mathrm{FC}=-0.27$; Adj. P Value $=0.01$; Pearson $\mathrm{r}-0.18$, Adj. P Value 0.04) and Cyclic GMP-AMP Synthase (CGAS) $(\log 2 \mathrm{FC}=-0.56$, Adj. P Value 7.89E-05; Pearson r -0.34, Adj. P Value 6.6E$5)$. Both genes encode proteins that activate the immune response to viruses $[26,27]$. 
(See figure on next page.)

Fig. 1 Gene expression differences between dermal fibroblast cell lines derived from the oldest ( $\geq 80$ years) and youngest ( $\leq 10$ years) age groups. a Volcano plot showing gene expression differences between oldest and youngest age groups. b KEGG pathways enriched in differentially expressed genes between the oldest and youngest age groups. $\mathbf{c}$ Heatmap of differentially expressed pattern recognition receptor genes between the oldest and youngest age groups. $\mathbf{d}$ Violin plots of the pattern recognition receptor genes that had an Adjusted PValue $<0.05$ and a log 2 FC $>1.0$ between the oldest and youngest age groups

To explore our findings further, we performed a differential gene expression analysis on the dermal fibroblast cell lines that had high ( $>75$ th percentile) and low $(<25$ th percentile) expression of TLR4 (Additional file 5: Suppl Table 4). Curiously, enrichment analysis of the 789 differentially expressed genes showed cell cycle (KEGG: hsa04110) to be the canonical pathway with the greatest enrichment (FDR 1.55E-06), similar to the enrichment of the differentially expressed genes between oldest and youngest groups (Fig. 1b,c, Additional file 6: Suppl Table 5). TLR4 is known to act via the adaptor molecule TRIF to regulate the expression of type I interferons. TLR activation of TRIF can also induce the cell cycle, an effect which is antagonized by type I interferons [28]. Our finding of both high levels of TLR4 and elevated cell cycle could thus imply changes in the expression of type I interferons.

\section{ACE2 expression increases with age}

We then examined whether the expression of $A C E 2$, the receptor for SARS-CoV-2, changes with age. ACE2 expression was detected in 35 of the 133 cell lines (26.3\%) and showed a marked increase in the $80+$ age group (Fig. 2b right). ACE2 expression was correlated with the expression of 19 of the 21 PRR genes (Fig. 2a and Additional file: Suppl Table 3a-c). Of note, ACE2 was expressed at much lower levels than TLR4, with variable expression in the 80 year and over age group. Whether the latter reflects the biological state of the individuals who donated the skin samples or is a consequence of ex vivo culture will require further study.

\section{Age-related interactions with SARS-CoV-2 proteins}

We also asked the question if the differentially expressed genes between the oldest and youngest age groups encode proteins that interact with SARS-CoV-2 (see "Methods" section). Our analysis revealed eleven differentially expressed genes between the oldest and youngest age groups that encode proteins known to interact with SARS-CoV-2 (Fig. 2d). Four of these genes (ADAM9, FBLN5, FAM8A1, CLIP4) have increased expression in the older compared to the younger age groups and are known to interact with four SARS-CoV-2 proteins (NsP9, Nsp13, M, and Orf8). Interestingly, the SARS-CoV-2 proteins to which they bind relate to lipid modifications and vesicle trafficking. Host interactions of Orf8 (endoplasmic reticulum quality control), M (ER structural morphology proteins), and NSp13 (golgins) may facilitate the dramatic reconfiguration of ER/Golgi trafficking during coronavirus infection [18]. Orf8 has also been suggested to promote immune evasion by downregulating antigen presentation in SARS-CoV-2 infected cells [29]. Whether age-related increases in the expression of host proteins that bind SARS-CoV-2 protein predispose to COVID19 disease or change its clinical course deserves further study.

\section{Discussion}

The COVID-19 (Coronavirus Disease-2019) pandemic is presenting unprecedented challenges to health care systems and governments worldwide. As of February 6, 2021 there have been 105,866,930 confirmed cases worldwide, resulting in 2,311,227 [30]. COVID-19 disease is caused by the novel Severe Acute Respiratory Syndrome Coronavirus 2 (SARS-CoV-2). SARS-CoV-2 is a single-stranded enveloped RNA virus, with viral entry depending upon binding of its spike protein to Angiotensin Converting Enzyme II (ACE2), a transmembrane protein present on the surface of multiple types of cells [31]. Infection of cells by SARS-CoV-2 disrupts cellular metabolism and compromises cellular survival by triggering apoptosis. Given the rapid spread of the virus and its associated mortality, there is a critical need to better understand the biology of the SARS-CoV-2 infection.

In this study, we used RNA-seq data from a large collection of dermal fibroblasts to demonstrate that PRR genes and $A C E 2$ vary with extremes of age. Further, we show that advanced age is associated with increased expression of several genes that encode proteins known to bind to SARS-CoV-2. Whether these gene expression differences contribute to the epidemiology of SARS$\mathrm{CoV}-2$ infection will require further study. Nevertheless, overexpression of PRR genes, TLR4 in particular, is an intriguing mechanism to explain the relationship between age and SARS-CoV-2 infection, and potentially the TLR-mediated cytokine storm that characterizes the morbidity and mortality in COVID-19 disease.

TLR4 has been previously suggested to have a role in the damaging responses that occurs during viral infections, acting via both PAMPs and DAMPs [9, 10]. 
a

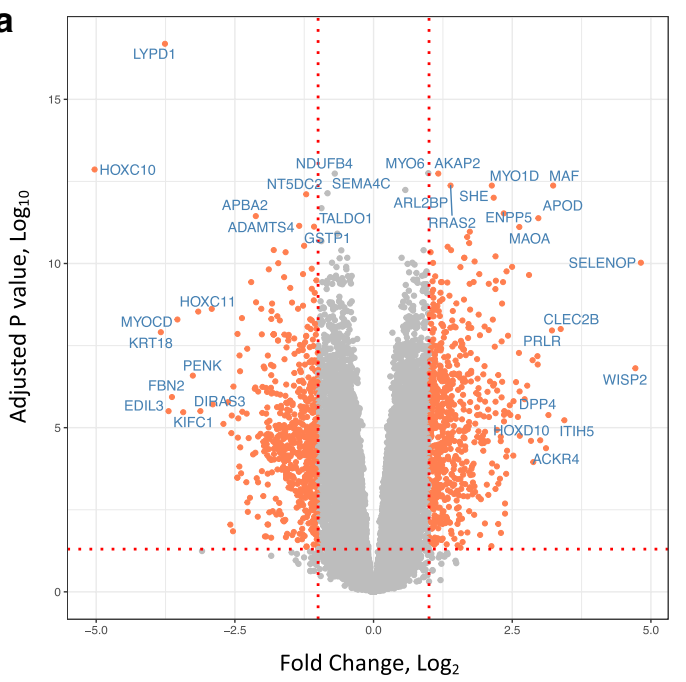

b

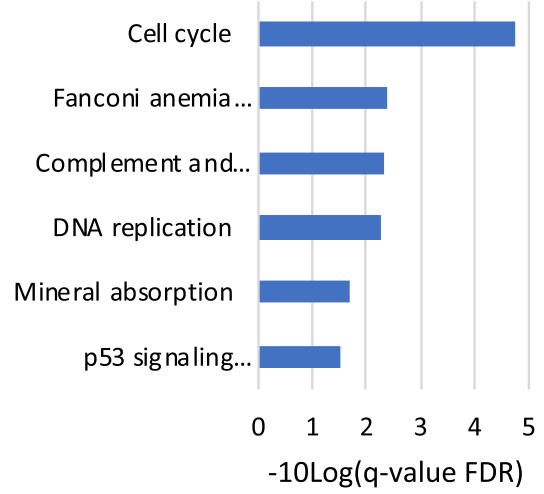

C

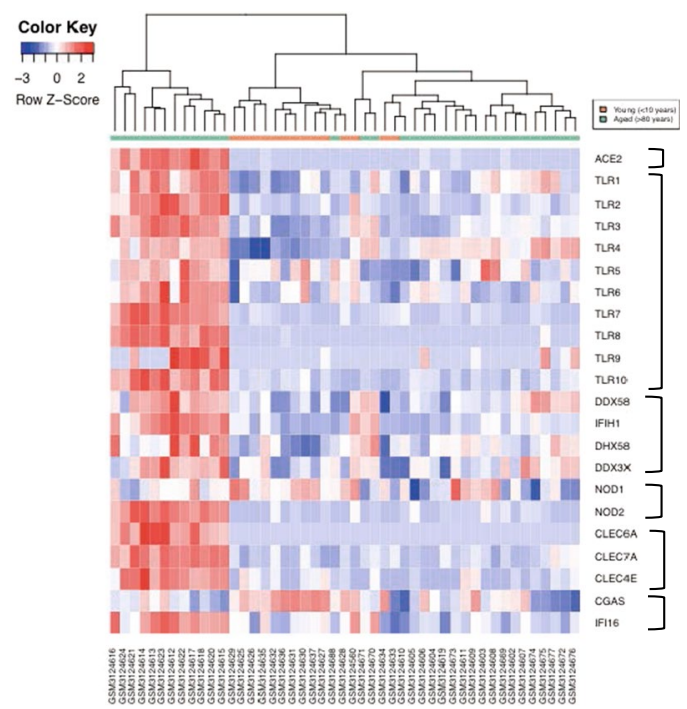

SARS-CoV-2 receptor

Toll-like receptors

RIG-I-like receptors

NOD-like receptors

C-type lectin receptors

Intracellular DNA sensors

d

TLR3

Adj.P.Val $=4.57 \mathrm{E}-06$

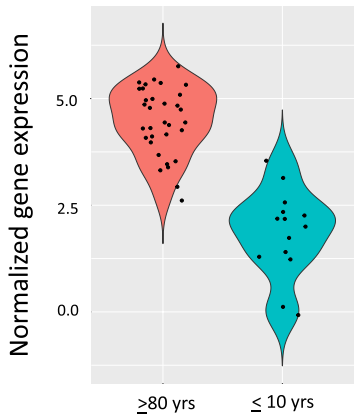

TLR4

Adj.P.Val $=5 \cdot 29 \mathrm{E}-08$

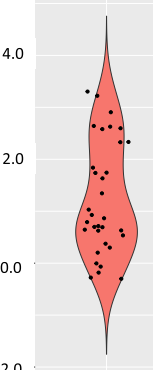

$\geq 80 \mathrm{yrs}$

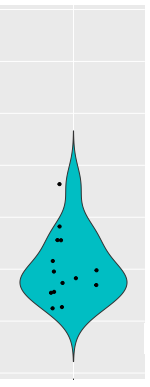

$\leq 10$ yrs
IFIH1

Adj.P.Val $=1.38 \mathrm{E}-04$

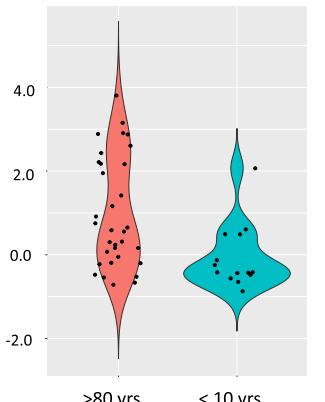

Age group 


\begin{abstract}
(See figure on next page.)
Fig. 2 Effect of age on the expression of pattern recognition receptor genes, enrichment results of high and low TLR4 expressors, and predicted interactions with SARS-CoV-2 proteins. a Correlation matrix comparing the relationships between age, ACE2 and 21 pattern recognition receptor genes. Pearson r, P values, and Confidence intervals of $r$ are provided in Additional file 3: Suppl Table 3a-c. Age refers to the age of the individual from which the dermal fibroblast cell line was derived. b Normalized gene counts for TLR3, TLR4, IHIF1 and ACE2 expressed as a function of age. $\mathbf{c}$ Enriched KEGG pathways in differentially expressed genes (absolute log2FC $>1.0$ and Adjusted P Value $<0.05$ ) between dermal fibroblast cell lines with high ( $>75$ th percentile) and low ( $<25$ th percentile) expression of TLR4. Based on differentially expressed genes with an absolute log2FC $>1.0$ and Adjusted P Value $<0.05$ ). $\mathbf{d}$ Protein-protein interactions linking differentially expressed genes between oldest ( $\geq 80$ years) and youngest ( $\leq 10$ years) age groups and SARS-CoV-2 proteins. SARS-CoV-2 viral proteins are represented at the center of each module, with interacting human host proteins represented with circles. Differentially expressed gene color is proportional to logFC. Physical interactions among host and viral proteins are noted as thin black lines. Four genes (ADAM9, FBLN5, FAM8A1, CLIP4) that encode proteins that interact with SARS-CoV-2 had increased expression in the oldest compared to youngest age groups (shades of red)
\end{abstract}

Diabetes, obesity and coronary artery disease are some of the conditions in which increased TLR4 expression has been reported [32, 33]. Notably, when blood from individuals with stable coronary artery disease and obese patients with atherosclerosis are stimulated with TLR ligands there is an increased cytokine response [34, 35]. Platelet TLR4 also has an important role in thrombosis [36], thus potentially linking toll-receptor expression to the hypercoagulability observed in COVID-19 patients [37]. Perhaps the best evidence that TLR4 has a role in SARS-CoV-2 infection is the observation that TLR4 mediated inflammatory signaling molecules are upregulated in peripheral blood mononuclear cells from COVID-19 patients, compared with healthy controls [38]. Among the most highly increased inflammatory mediators in severe/critically ill patients is S100A9, an alarmin and TLR4 ligand. Recombinant S2 and nucleocapsid proteins of SARS-CoV-2 significantly increased pro-inflammatory cytokines/chemokines and S100A9 in human primary PBMCs [38]. Considered together, changes in the expression of TLRs and other PRRs could have a key role in mediating the age-related inflammatory response during SARS CoV-2 infection.

We recognize that dermal fibroblasts are not the primary entry site of SARS-CoV-2 into the human body. Nevertheless, the SARS-CoV-2 virus has been found at sites outside the respiratory tract [39]; $A C E 2$ is highly expressed in the granulosum of the skin [40], and fibroblast have been used to investigate host antiviral defenses during other Coronavirus infections [41]. The pressing question is how closely fibroblasts simulate the biology of cells within the respiratory system, and if they could be a useful model for studying SARS-CoV-2 infection. Limited data suggests there are biological similarities between the age-related changes that occur in dermal fibroblast and within the lung. Chow et al. [42] analyzed 578 lung RNA seq transcriptomes from donors of varying ages (2170 years old) available from the Genotype-Tissue Expression (GTEx) project [43]. After correcting for sex, age, smoking status and Hardy scale, age was an independent predictor of $A C E 2$ expression, with increasing age associated with higher expression of ACE2-similar to that observed in our dermal fibroblast model. Interestingly, Cell Cycle was the most highly enriched DAVID gene ontology pathway in the age-down gene expression group in the GTEx dataset and the most highly enriched KEGG pathway in our $\leq 10$ and $\geq 80$ years age group comparison. Further, the dermal fibroblast model was similar to the GTEx lung dataset in that it predicted three of the four age-related protein-protein SARS-CoV-2 interactions (Nsp9, Nsp13, Orf8).

To explore this further, we tested the hypothesis that PRR gene expression was associated with age using the lung transcriptome data in the GTEx dataset. To do so, we queried Supplementary Table 6 of the Chow et al. manuscript [42] for the 21 PRR genes used in our study. We found no association between age and PRR gene expression using the GTEx data set. Nevertheless, an important caveat of the analysis is that the donors in the GTEx dataset were between 21-70 years of age. It thus does not include samples at the extremes of age which are likely to be biologically different.

Our study does have other limitations. Foremost, is that health information was not available for the individuals donating skin samples to the dermal fibroblast collection. Although, the skin samples are reported to be from "apparently healthy individuals", we believe it is unlikely that individuals in the oldest age group were completely free of chronic diseases. Another limitation was that minority groups are inadequately represented in the collection. The dermal fibroblast collection includes samples from one American Indian $(<1 \%)$, one Hispanic $(<1 \%)$, two Asians (1.5\%), and nine Blacks (6.7\%)-way too few to draw any meaningful conclusions on the ethnic groups that have been the hardest hit by the COVID19 pandemic.

Finally, as the scientific community continues its research on the COVID-19 pandemic, the dermal fibroblast model provides another potential tool for investigating SARS-CoV-2 biology. The strength of the 
a

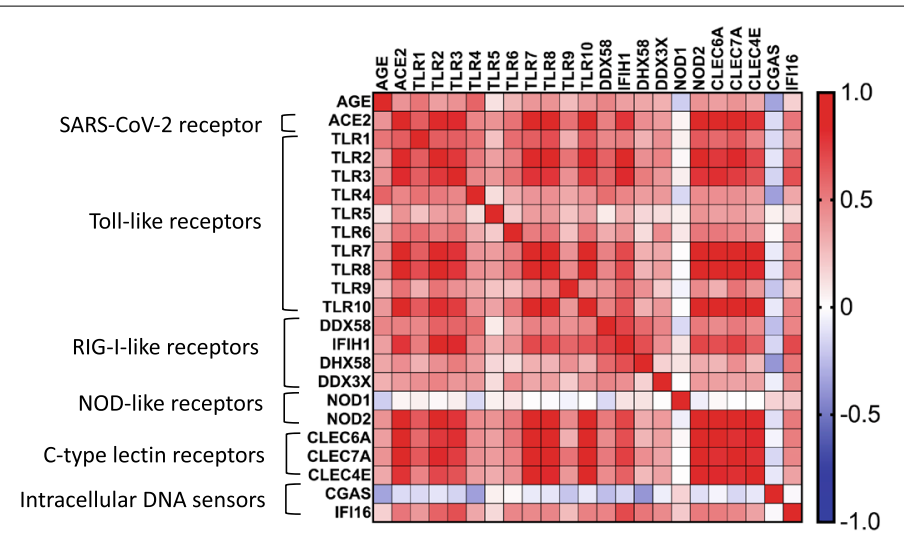

b

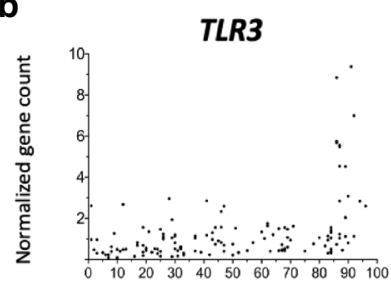

Age (years)

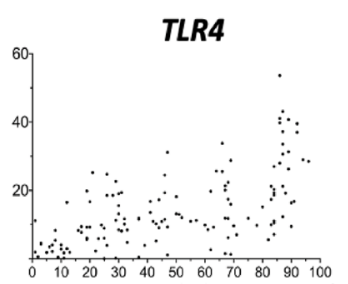

Age (years)

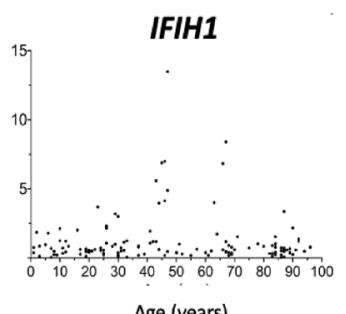

Age (years)

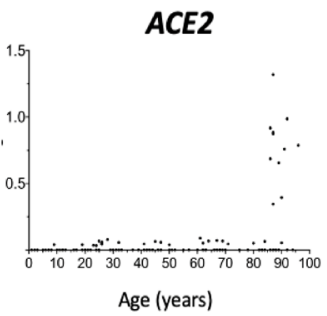

d

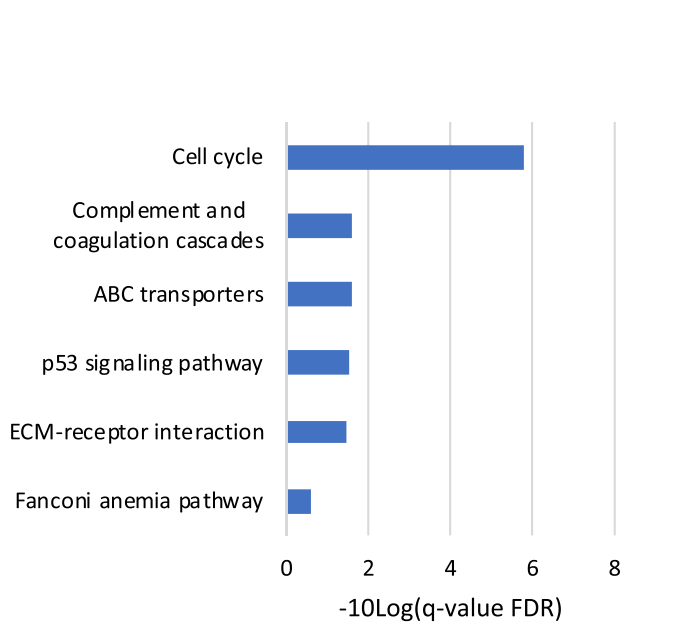

c
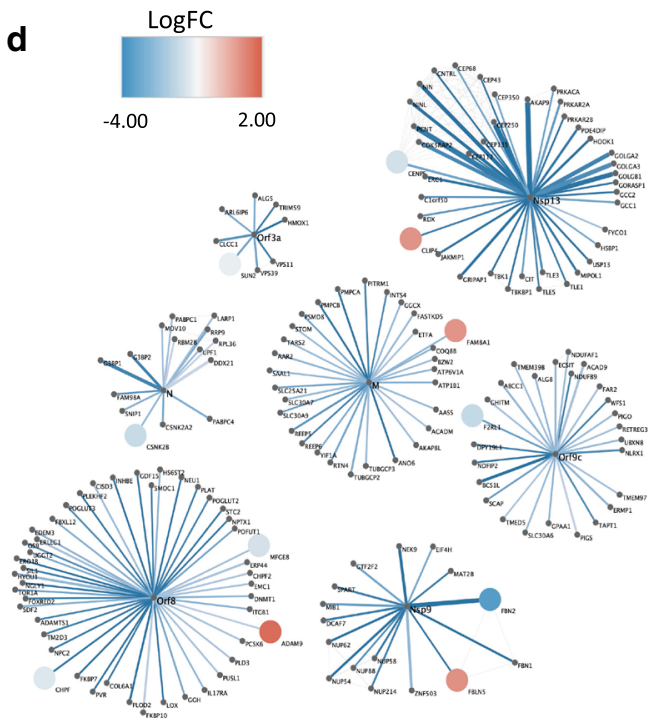

dermal fibroblast model is that skin samples can be easily obtained from donors of different ages, sex, and ethnicities, and those with varying comorbidities such a high blood pressure and diabetes, and from smokers and nonsmokers. This approach could be especially valuable in children were invasive procedures to collect tissue is less acceptable and post-mortem collection of tissue is less common. Such a model would also have an advantage over transfection models as these cells would not only have increased expression of ACE2 and TLR4, but also have an aged transcriptome which could be important for the infectivity and outcome of the SARS-CoV-2 infection. 
The critical role PRRs play in mediating host-pathogen interactions, and their increased expression in some comorbidities associated with poor COVID-19 outcomes, make them a potential target for developing tools to predict risk for and outcomes of SARS-CoV-2 infection at both the individual and population levels.

\section{Conclusions}

Using a large dataset of genome-wide RNA-seq profiles derived from human dermal fibroblasts we show that expression of PRR genes and ACE2, the receptor for SARS-CoV-2 vary with extremes of age. Advanced age was also associated with increased expression of several genes that encode proteins which interact with SARS$\mathrm{CoV}-2$. Given that PRRs function as a critical interface between the host and invading pathogens, further research is needed to better understand how changes in PRR expression affects the susceptibility to and outcome of SARS-CoV-2 infection.

\begin{abstract}
Abbreviations
ACE2: Angiotensin Converting Enzyme II; CGAS: Cyclic GMP-AMP Synthase; CLEC4E: C-Type Lectin Domain Family 4 Member E; CLEC6A: C-Type Lectin Domain Containing 6A; CLEC7A: C-Type Lectin Domain Containing 7A; CLR: C-type Lectin Receptor; COVID-19: Coronavirus Disease-2019; DAMP: DamageAssociated Molecular Pattern; DDX3X: DEAD-Box Helicase 3 X-Linked; DDX58: DExD/H-Box Helicase 58; DHX58: DExH-Box Helicase 58; IFI16: Interferon Gamma Inducible Protein 16; IFIH1: Interferon Induced With Helicase C Domain 1; NLR: NOD-Like Receptor; NOD1: Nucleotide Binding Oligomerization Domain Containing 1; NOD2: Nucleotide Binding Oligomerization Domain Containing 2; PAMP: Pathogen-Associated Molecular Pattern; PRR: Pattern Recognition Receptor; SARS-CoV-2: Severe Acute Respiratory Syndrome Coronavirus 2; TLR: Toll Like Receptor.
\end{abstract}

\section{Supplementary Information}

The online version contains supplementary material available at https://doi. org/10.1186/s12920-021-00970-7.

Additional file 1. Supplementary Table 1. Gene expression analysis between the oldest ( $\geq 80$ years) and youngest $(\leq 10)$ age groups: a) Differentially expressed genes with an Adjusted P Value $<0.05$ and Absolute FC $>1.0, b)$ ACE2 and PRR genes

Additional file 2. Supplementary Table 2. ToppGene enrichment results for differentially expressed genes between the oldest and youngest age groups (filtered to show KEGG pathway results)

Additional file 3. Supplementary Table 3. a) Pearson r, b) P values, and c) Confidence intervals of $r$ for the correlation matrix shown in Fig. $2 a$

Additional file 4. Supplementary Figure 1. Normalized gene counts for the ten Toll-like receptors expressed as a function of age

Additional file 5. Supplementary Table 4. Differentially expressed genes between TLR4 high vs low expressors

Additional file 6. Supplementary Table 5. ToppGene enrichment results for differentially expressed genes between TLR4 high and low expressors (filtered to show KEGG pathway results)

\section{Acknowledgements}

Not applicable.

\section{Authors' contributions}

SB designed and conceived the research. SB and KF analyzed the data. All authors contributed to the content of the manuscript and read and approved the final version.

\section{Funding}

Not applicable.

\section{Availability of data and material}

The original dataset can be downloaded from NCBI GEO repository (accession number GSE 113,957).

\section{Declarations}

Ethics approval and consent to participate

Not applicable.

\section{Consent for publication}

Not applicable.

\section{Competing interests}

The authors declare that they have no competing interests.

\section{Author details}

'Rady Children's Hospital, Rady Children's Hospital, University of California San Diego, 3030 Children's Way, San Diego, CA 92123, USA. ²Department of Surgery, University of California San Diego, La Jolla, CA, USA. ${ }^{3}$ Center for Investigations of Health and Education Disparities, University of California San Diego, La Jolla, CA, USA. ${ }^{4}$ Center for Computational Biology and Bioinformatics, University of California San Diego, La Jolla, CA, USA. ${ }^{5}$ Naval Medical Center San Diego, San Diego, CA, USA. ${ }^{6}$ Timpanogos Regional Hospital, Orem, UT, USA. ${ }^{7}$ VA Medical Center, San Diego, CA, USA. ${ }^{8}$ Department of Anesthesia, University of California San Francisco, San Francisco, CA, USA.

Received: 31 August 2020 Accepted: 28 April 2021

Published online: 24 May 2021

\section{References}

1. Arons MM, Hatfield KM, Reddy SC, Kimball A, James A, Jacobs JR, et al. Presymptomatic SARS-CoV-2 infections and transmission in a skilled nursing facility. N Engl J Med. 2020;382(22):2081-90.

2. CDC. Preliminary Estimates of the Prevalence of Selected Underlying Health Conditions Among Patients with Coronavirus Disease 2019 United States, February 12-March 28, 2020. MMWR Morb Mortal Wkly Rep. 2020;69(13):382-6.

3. Verity R, Okell LC, Dorigatti I, Winskill P, Whittaker C, Imai N, et al. Estimates of the severity of coronavirus disease 2019: a model-based analysis. Lancet Infect Dis. 2020;20(6):669-77.

4. Williamson EJ, Walker AJ, Bhaskaran K, Bacon S, Bates C, Morton CE, Curtis $\mathrm{HJ}$, et al. Factors associated with COVID-19-related death using OpenSAFELY. Nature. 2020;584(7821):430-6.

5. Brubaker SW, Bonham KS, Zanoni I, Kagan JC. Innate immune pattern recognition: a cell biological perspective. Annu Rev Immunol. 2015;33:257-90.

6. Gong T, Liu L, Jiang W, Zhou R. DAMP-sensing receptors in sterile inflammation and inflammatory diseases. Nat Rev Immunol. 2020;20(2):95-112.

7. Doyle SL, O'Neill LA. Toll-like receptors: from the discovery of NFkappaB to new insights into transcriptional regulations in innate immunity. Biochem Pharmacol. 2006;72(9):1102-13.

8. Tartey S, Takeuchi O. Pathogen recognition and Toll-like receptor targeted therapeutics in innate immune cells. Int Rev Immunol. 2017;36(2):57-73.

9. Olejnik J, Hume AJ, Muhlberger E. Toll-like receptor 4 in acute viral infection: too much of a good thing. PLoS Pathog. 2018;14(12):e1007390.

10. Saghazadeh A, Rezaei N. Implications of Toll-like receptors in Ebola infection. Expert Opin Ther Targets. 2017;21(4):415-25.

11. Zhang Z, Wang C, Liu Z, Zou G, Li J, Lu M. Host genetic determinants of hepatitis B virus infection. Front Genet. 2019;10:696. 
12. Arpaia N, Barton GM. Toll-like receptors: key players in antiviral immunity. Curr Opin Virol. 2011;1 (6):447-54.

13. GREIN-iLINCS. [Available from: http://www.lincs.org/apps/grein/.

14. Mahi NA, Najafabadi MF, Pilarczyk M, Kouril M, Medvedovic M. GREIN: an interactive web platform for re-analyzing GEO RNA-seq data. Sci Rep. 2019;9(1):7580.

15. Ritchie ME, Phipson B, Wu D, Hu Y, Law CW, Shi W, et al. limma powers differential expression analyses for RNA-sequencing and microarray studies. Nucleic Acids Res. 2015;43(7):e47.

16. Law CW, Chen Y, Shi W, Smyth GK. voom: Precision weights unlock linear model analysis tools for RNA-seq read counts. Genome Biol. 2014;15(2):R29.

17. Chen J, Bardes EE, Aronow BJ, Jegga AG. ToppGene Suite for gene list enrichment analysis and candidate gene prioritization. Nucleic Acids Res. 2009;37(Web Server issue):W305-11.

18. Gordon DE, Jang GM, Bouhaddou M, Xu J, Obernier K, White KM, et al. A SARS-CoV-2 protein interaction map reveals targets for drug repurposing. Nature. 2020.

19. Shannon P, Markiel A, Ozier O, Baliga NS, Wang JT, Ramage D, et al. Cytoscape: a software environment for integrated models of biomolecular interaction networks. Genome Res. 2003;13(11):2498-504.

20. Pillich RT, Chen J, Rynkov V, Welker D, Pratt D. NDEx: a community resource for sharing and publishing of biological networks. Methods Mol Biol. 2017:1558:271-301.

21. Orioli D, Dellambra E. Epigenetic Regulation of Skin Cells in Natural Aging and Premature Aging Diseases. Cells. 2018;7(12).

22. Glass D, Vinuela A, Davies MN, Ramasamy A, Parts L, Knowles D, et al. Gene expression changes with age in skin, adipose tissue, blood and brain. Genome Biol. 2013;14(7):R75.

23. Gronniger E, Weber B, Heil O, Peters N, Stab F, Wenck H, et al. Aging and chronic sun exposure cause distinct epigenetic changes in human skin. PLoS Genet. 2010;6(5):e1000971.

24. Jung M, Jin SG, Zhang X, Xiong W, Gogoshin G, Rodin AS, et al. Longitudinal epigenetic and gene expression profiles analyzed by threecomponent analysis reveal down-regulation of genes involved in protein translation in human aging. Nucleic Acids Res. 2015;43(15):e100.

25. Fleischer JG, Schulte R, Tsai HH, Tyagi S, Ibarra A, Shokhirev MN, et al. Predicting age from the transcriptome of human dermal fibroblasts. Genome Biol. 2018;19(1):221.

26. $\mathrm{Ni} \mathrm{G}, \mathrm{Ma}$ Z, Damania B. CGAS and STING: At the intersection of DNA and RNA virus-sensing networks. PLoS Pathog. 2018;14(8):e1007148.

27. Coutermarsh-Ott S, Eden K, Allen IC. Beyond the inflammasome: regulatory NOD-like receptor modulation of the host immune response following virus exposure. J Gen Virol. 2016;97(4):825-38.

28. Hasan UA, Caux C, Perrot I, Doffin AC, Menetrier-Caux C, Trinchieri G, et al. Cell proliferation and survival induced by Toll-like receptors is antagonized by type I IFNs. Proc Natl Acad Sci USA. 2007;104(19):8047-52.

29. Zhang Y, al. e. The ORF8 protein of SARS-CoV-2 mediates immune evasion through potentially down regulating MHC-1. bioRxiv2020.
30. Dong E, Du H, Gardner L. An interactive web-based dashboard to track COVID-19 in real time. Lancet Infect Dis. 2020;20(5):533-4.

31. Hoffmann M, Kleine-Weber H, Schroeder S, Kruger N, Herrler T, Erichsen $\mathrm{S}$, et al. SARS-CoV-2 cell entry depends on ACE2 and TMPRSS2 and is blocked by a clinically proven protease inhibitor. Cell. 2020;181 (2):271-80.

32. Freedman JE, Larson MG, Tanriverdi K, O'Donnell CJ, Morin K, Hakanson $\mathrm{AS}$, et al. Relation of platelet and leukocyte inflammatory transcripts to body mass index in the Framingham heart study. Circulation. 2010:122(2):119-29.

33. Shao L, Zhang P, Zhang Y, Lu Q, Ma A. TLR3 and TLR4 as potential clinically biomarkers of cardiovascular risk in coronary artery disease (CAD) patients. Heart Vessels. 2014;29(5):690-8.

34. Elsenberg EH, Sels JE, Hillaert MA, Schoneveld AH, van den Dungen NA, van Holten TC, et al. Increased cytokine response after toll-like receptor stimulation in patients with stable coronary artery disease. Atherosclerosis. 2013;231(2):346-51.

35. Scholtes VP, Versteeg D, de Vries JP, Hoefer IE, Schoneveld AH, Stella PR, et al. Toll-like receptor 2 and 4 stimulation elicits an enhanced inflammatory response in human obese patients with atherosclerosis. Clin Sci (Lond). 2011;121(5):205-14.

36. Schattner M. Platelet TLR4 at the crossroads of thrombosis and the innate immune response. J Leukoc Biol. 2019;105(5):873-80

37. Bikdeli B, Madhavan MV, Jimenez D, Chuich T, Dreyfus I, Driggin E, et al. COVID-19 and Thrombotic or Thromboembolic Disease: Implications for Prevention, Antithrombotic Therapy, and Follow-up. J Am Coll Cardiol. 2020.

38. Sohn KM, Lee SG, Kim HJ, Cheon S, Jeong H, Lee J, et al. COVID-19 patients upregulate toll-like receptor 4-mediated inflammatory signaling that mimics bacterial sepsis. J Korean Med Sci. 2020;35(38):e343.

39. Xia J, Tong J, Liu M, Shen Y, Guo D. Evaluation of coronavirus in tears and conjunctival secretions of patients with SARS-CoV-2 infection. J Med Virol. 2020;92(6):589-94.

40. Sun Y, Zhou R, Zhang H, Rong L, Zhou W, Liang Y, et al. Skin is a potential host of SARS-CoV-2: A clinical, single-cell transcriptome-profiling and histologic study. J Am Acad Dermatol. 2020;83(6):1755-7.

41. Deng X, Baker SC. An "Old" protein with a new story: Coronavirus endoribonuclease is important for evading host antiviral defenses. Virology. 2018:517:157-63.

42. Chow RD, Majety M, Chen S. The aging transcriptome and cellular landscape of the human lung in relation to SARS-CoV-2. Nat Commun. 2021;12(1):4.

43. Consortium G. The Genotype-Tissue Expression (GTEx) project. Nat Genet. 2013;45(6):580-5.

\section{Publisher's Note}

Springer Nature remains neutral with regard to jurisdictional claims in published maps and institutional affiliations.
Ready to submit your research? Choose BMC and benefit from:

- fast, convenient online submission

- thorough peer review by experienced researchers in your field

- rapid publication on acceptance

- support for research data, including large and complex data types

- gold Open Access which fosters wider collaboration and increased citations

- maximum visibility for your research: over $100 \mathrm{M}$ website views per year

At BMC, research is always in progress.

Learn more biomedcentral.com/submissions 